\title{
Controle autonômico da freqüência cardíaca em Spilotes pullatus (Colubridae) como determinante das respostas de medo
}

[Autonomic control of heart rate in Spilotes pullatus (Colubridae) as determinant of fear responses]

J.G.P. Cruz, L. Junkes

Departamento de Ciências Naturais - CCEN-FURB

Rua Antônio da Veiga, 140

89010-971 - Blumenau, SC

\section{RESUMO}

Neste estudo, investigaram-se as modificações fisiológicas em Spilotes pullatus, como determinantes das respostas ao medo, obtidas pela exposição ao eletrocardiograma. Foram utilizadas quatro serpentes com média de peso de $0,5 \pm 0,06 \mathrm{~kg}$. Dois minutos após a fixação dos eletrodos, as serpentes apresentavam-se imóveis, com redução significativa na freqüência cardíaca e aumento nos intervalos PQ, QRS, RR e RT $(\mathrm{P}<0,05)$. Os resultados sugerem que a presença de sintomas de medo pode gerar riscos à saúde desses animais.

Palavras-chave: serpente, caninana, bradicardia, eletrocardiograma, medo

\begin{abstract}
This study investigated electrocardiographic changes in Spilotes pullatus using four snakes weighing $0.5 \pm 0.06 \mathrm{~kg}$. Two minutes after that electrocardiogram apparatus had been fixed in Spilotes pullatus, snakes were immobile and there was a significant reduction of the heart rate and an increase in $P Q, Q R S, R R$, and $R T$ intervals $(P<0.05)$. Results suggest that the presence of fear symptoms may represent a risk to the health of snakes.
\end{abstract}

Keywords: snake, caninana, bradycardia, electrocardiogram, fear

\section{INTRODUÇÃO}

O medo ou a ansiedade é importante para a sobrevivência da espécie e está relacionado com a interação do organismo com o meio ambiente. Sua função é proteger o indivíduo e a espécie contra os perigos que envolvem o animal, principalmente contra os predadores. As respostas comportamentais de defesa são acompanhadas por várias alterações autonômicas e visam capacitar os animais a um melhor desempenho nas tarefas de se protegerem e evitarem as situações de perigo e ameaça (Guimarães-Costa et al., 2007). Tanto o medo como a ansiedade podem ter suas origens nas reações de defesa do animal; ambas podem gerar respostas psicofisiológicas semelhantes, o que

Recebido em 15 de outubro de 2007

Aceito em 20 de setembro de 2008

E-mail: jgcruz@furb.br leva a supor a existência de mecanismos neurais comuns (Graeff, 1993).

A resposta ao medo/ansiedade é caracterizada por imobilidade e aumento da pressão sangüínea e dos batimentos cardíacos, além de outros sinais autonômicos. Essas respostas são mediadas pelo sistema nervoso autônomo simpático, pois podem ser abolidas pelo bloqueio farmacológico dos gânglios autonômicos por antagonistas dos receptores $\alpha$-adrenérgicos (Carrive, 2002). Esse controle está relacionado com os efeitos mediados pelos barorreflexos, com a redução da atividade parassimpática e aumento da simpática sobre o coração, com o objetivo de manter a pressão arterial média elevada, permitindo que o animal redirecione suas atividades, podendo passar da imobilidade para movimentos (Nijsen et al., 2000; Choi et al., 2005). 
O objetivo do presente trabalho foi determinar a freqüência cardíaca e as variações eletrocardiográficas da caninana (Spilotes pullatus, Serpentes: Colubridae), quando é submetida, pela primeira vez, ao medo/ansiedade provocado pela avaliação clínica.

\section{MATERIAL E MÉTODOS}

$\mathrm{O}$ experimento foi realizado no período matutino entre 8 e 11 horas, com quatro fêmeas adultas de $S$. pullatus, com média de peso de $0,5 \pm 0,06 \mathrm{~kg}$ e comprimento de $1,69 \pm 0,1 \mathrm{~m}$, obtidas no serpentário da Universidade Regional de Blumenau, SC ${ }^{1}$. A temperatura corporal e as variações cardíacas foram determinadas no laboratório do serpentário, com temperatura ambiente de $28,3 \pm 2^{\circ} \mathrm{C}$. Nos animais, colocados em decúbito ventral, fixaram-se um eletrodo de referência, lateralmente, no lado direito e acima do coração, e um eletrodo ativo, no lado esquerdo, abaixo do coração e de forma análoga à derivação II. Após avaliação da temperatura da cloaca $^{2}$, realizou-se o eletrocardiograma ${ }^{3}$, para análise dos intervalos PQ, QRS, RT, RR e obtenção da freqüência cardíaca, aos 30 e 120 segundos. Para as comparações entre os intervalos e a freqüência cardíaca, foi utilizado o teste $\mathrm{t}$ pareado, expressos com média e erro-padrão da média $(\mathrm{P}<0,05)$.

\section{RESULTADOS}

Os animais, dois minutos após a fixação dos eletrodos ao seu corpo, apresentavam-se totalmente imóveis, com a temperatura corporal de $31,1 \pm 0,3^{\circ} \mathrm{C}$.

No eletrocardiograma, observaram-se arritmia e aumento da atividade do nervo vago e/ou diminuição do simpático, com redução significativa dos batimentos cardíacos de $64 \pm 3$ para $23 \pm 2$ por minuto.

Diferenças significativas também foram observadas na duração dos intervalos PQ, QRS, RT e RR, com aumento dessas medidas (Fig. 1). O intervalo PQ aumentou de forma significativa de $0,13 \pm 0,01$ para $0,24 \pm 0,04$ segundos $(\mathrm{P}<0,05), \quad$ o $\quad$ QRS de $0,07 \pm 0,005$ para $0,11 \pm 0,006$ segundos $(\mathrm{P}<0,01)$, o RT de $0,38 \pm 0,01$ para $0,55 \pm 0,05$ segundos $(\mathrm{P}<0,001)$ e o $\mathrm{RR}$ de $0,97 \pm 0,04$ para $2,58 \pm 0,22$ segundos $(\mathrm{P}<0,001)$.
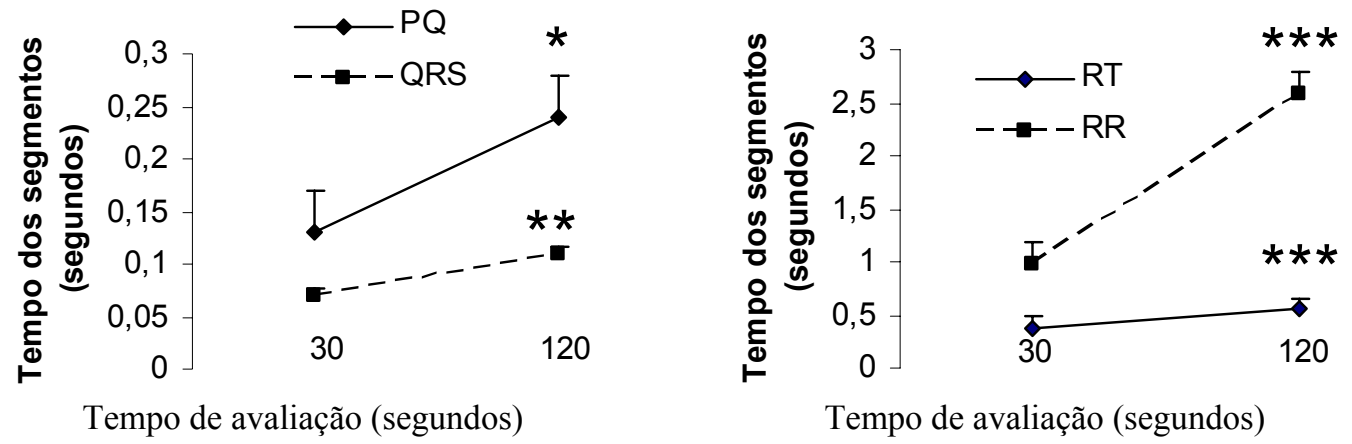

Figura 1. Tempo dos segmentos PQ, QRS, RT e RR observados em Spilotes pullatus, representados pelas médias e erros-padrão das médias de quatro animais. ${ }^{*} \mathrm{P}<0,05 ; * * \mathrm{P}<0,01 ; * * * \mathrm{P}<0,001$.

O mesmo padrão eletrocardiográfico foi observado em todos os animais, com imobilidade ao serem fixados os eletrodos e retorno à atividade após a sua retirada. Esse mesmo procedimento, quatro horas após, demonstrou que, apesar de os animais continuarem imóveis após a fixação dos eletrodos, a freqüência cardíaca manteve-se dentro dos parâmetros iniciais durante os cinco minutos em que esses estiveram conectados ao eletrocardiógrafo (dados não mostrados). Isso sugere que os estímulos apresentados pelo ambiente e identificados pelo animal são responsáveis pelas respostas apresentadas.

\section{DISCUSSÃO}

Nos vertebrados, a percepção dos estímulos nocivos provenientes do ambiente, por meio do sistema sensorial (olfato, visão, tato, dor), permite a ativação de respostas comportamentais e fisiológicas adequadas à proteção, adaptando o organismo a diferentes contextos. 
A simples fixação de Spilotes pullatus aos eletrodos induz uma resposta de medo/ansiedade, caracterizada pela imobilidade comportamental e ativação do sistema nervoso parassimpático e/ou diminuição do simpático, com redução da freqüência cardíaca. A arritmia sinusal observada durante a bradicardia resulta dos reflexos cardiovasculares, responsáveis pelas alterações nas intensidades dos sinais neurais simpáticos e parassimpáticos para o nodo sinusal.

Além da freqüência cardíaca, outro fator que pode demonstrar ativação do sistema nervoso parassimpático e/ou diminuição do simpático é o intervalo PQ e QRS (Croiset et al., 1994; Nijsen et al., 1998). O intervalo PQ define o intervalo entre o início da onda $\mathrm{P}$ e o início do complexo QRS, estabelecendo uma relação temporal na condução atrioventricular, influenciada pelo sistema nervoso autônomo. $\mathrm{O}$ aumento do intervalo PQ e QRS é causado pelo aumento na atividade vagal e/ou decréscimo da atividade simpática (Penz et al., 1992; Croiset et al., 1994). Em geral, o estresse induz a ativação simpática (Korte et al., 1992), mas pode aumentar o intervalo $P Q$ e QRS mediante ativação do sistema vagal, como observado na serpente estudada. O aumento dos intervalos RT e RR está em conformidade com a ativação do nervo vago sobre a propagação dos sinais elétricos sobre a massa cardíaca ventricular e a redução na freqüência cardíaca. Isso sugere que a ativação do sistema simpático e parassimpático estaria relacionada com a modulação das funções cardíacas durante o estado de medo/ansiedade (Nijsen et al., 1998).

O sistema comportamental de Spilotes pullatus é típico dos vertebrados em muitas de suas estratégias para enfrentar o estresse provocado pelas condições adversas do ambiente. Naquelas em que o medo/ansiedade permite uma possível solução, há ativação do sistema cardiovascular com taquicardia e aumento da pressão arterial. Essas estratégias fisiológicas permitem aos animais enfrentarem com o máximo de eficiência as condições ambientais adversas. Entretanto, alguns estímulos nocivos do ambiente podem ser de tal magnitude que, aparentemente, o sistema nervoso fica incapacitado de gerar uma resposta eficiente, podendo levar o organismo ao desfalecimento e à morte (síncope vagal) (Grubb et al., 1992; DeToledo, 1999). Nessa situação, observa-se imobilidade comportamental e bradicardia. Esse comportamento deve ser etologicamente estudado, para 0 melhor entendimento neurobiológico do medo/ansiedade. Quando houver necessidade de intervenções clínicas na espécie de serpente estudada, modificações fisiológicas podem gerar riscos à saúde do animal.

\section{REFERÊNCIAS BIBLIOGRÁFICAS}

CARRIVE, P. Cardiovascular and behavioural components of conditioned fear to context after ganglionic and alpha-adrenergic blockade. Auton. Neurosc., v.98, p.90-93, 2002.

CHOI, E.A.; SAMUEL, L.; DANIEL, M.L. et al. Expression of cardiovascular and behavioral components of conditioned fear to context in T4 spinally transected rats. Auton. Neurosc.: Basic Clin., v.120, p.26-34, 2005.

CROISET, G.; RAATS, C.J.L.; NIJSEN, M.J.M.A. et al. Differential effects of cholinergic and adrenergic agents on P-R and R-R intervals in rat ECG. Neurosc. Res. Commun., v.14, p.7584, 1994.

DeTOLEDO, J.C. Convulsive syncope following placement of sphenoidal electrodes. Brain Develop., v.21, p.213-215, 1999.

GRAEFF, F.G. Role of 5-HT in defensive behavior and anxiety. Rev. Neurosc., v.4, p.181211, 1993.

GUIMARÃES-COSTA， R.; GUIMARÃESCOSTA, M.B.; PIPPA-GADIOLI, L. et al. Innate defensive behaviour and panic-like reactions evoked by rodents during aggressive encounters with Brazilian constrictor snakes in a complex labyrinth: Behavioural validation of a new model to study affective and agonistic reactions in a prey versus predator paradigm. $J$. Neurosc. Methods, v.165, p.25-37, 2007.

GRUBB, B.P.; TEMESY-ARMOS, P.; MOORE, J. et al. Head-upright tilt-table testing in evaluation and management of the malignant vasovagal syndrome. Am. J. Cardiol., v.69, p.904-908, 1992.

KORTE, S.M.; BUWALDA, B.; BOUWS, G.A.H. et al. Conditioned neuroendocrine and cardiovascular stress responsiveness accompanying behavioral passivity and activity in aged and in young rats. Physiol. Behav., v.51, p.815-822, 1992. 
NIJSEN, M.J.M.A.; CROISET, G.; DIAMANT, $M$. et al.Vagal activation in novelty-induce tachycardia during the light phase in the rat. Physiol. Behav., v.63, p.233-239, 1998.

NIJSEN, M.J.M.A.; CROISET, G.; DIAMANT, M. et al. Conditioned fear-induced tachycardia in the rat; vagal involvement. Eur. J. Pharmacol., v.350, p.211-222, 1998.
NIJSEN, M.J.; CROISET, G.; DIAMANT, M. et al. Endogenous corticotropin-releasing hormone inhibits conditionede-fear-induced vagal activation in the rat. Eur. J. Pharmacol., v.389, p.89-98, 2000.

PENZ, W.; PUGSLEU, M.; HSIEH, M.Z. et al. A new ECG measure (RSh) for detecting possible sodium channel blockade in vivo in rats. J. Pharmacol. Methods, v.27, p.51-58, 1992. 